\title{
Photospheric flows around a quiescent filament
}

\author{
S. Rondi ${ }^{1}$, Th. Roudier ${ }^{1}$, G. Molodij ${ }^{2}$, V. Bommier ${ }^{3}$, S. Keil $^{5}$, P. Sütterlin ${ }^{4}$, J. M. Malherbe ${ }^{2}$, N. Meunier ${ }^{1}$, \\ B. Schmieder ${ }^{2}$, and P. Maloney ${ }^{5}$ \\ ${ }^{1}$ Laboratoire d'Astrophysique de l'Observatoire Midi-Pyrénées, Université Paul Sabatier Toulouse III, CNRS, 57 Avenue d'Azeirex, \\ BP 826, 65008 Tarbes Cedex, France \\ e-mail: roudier@ast.obs-mip. fr \\ 2 LESIA, Observatoire de Paris, Section de Meudon, 92195 Meudon, France \\ 3 LERMA, Observatoire de Paris, Section de Meudon, 92195 Meudon, France \\ 4 Sterrekundig Instituut Utrecht, Postbus 80 000, 3508 TA Utrecht, The Netherlands \\ 5 National Solar Observatory, Sacramento Peak, Sunspot, NM 88349, USA
}

Received 27 October 2006 / Accepted 1 March 2007

\section{ABSTRACT}

\begin{abstract}
Context. The horizontal photospheric flows below and around a filament are one of the components in the formation and evolution of filaments. Few studies exist because they require multiwalength time sequences at high spatial resolution.

Aims. Our objective is to measure the horizontal photospheric flows associated with the evolution and eruption of a filament. Methods. We present observations obtained in 2004 during the international JOP 178 campaign which involved eleven instruments both in space and at ground based observatories. We use TRACE WL, DOT and DST observation to derive flow maps which are then coaligned with intensity images and with the vector magnetic field map obtained with THEMIS/MTR.

Results. Several supergranulation cells cross the Polarity Inversion Line (PIL) and can transport magnetic flux through the PIL, in particular parasitic polarities. We present a detailed example of the formation of a secondary magnetic dip at the location of a filament footpoint. Large-scale converging flows, which could exist along the filament channel and contribute to its formation, are not observed. Before the filament's eruptive phase, we observe both parasitic and normal polarities being swept by a continuously diverging horizontal flow located in the filament gap. The disappearance of the filament initiates in this gap. Such purely horizontal motions could lead to destabilization of the filament and could trigger the sudden filament disappearance.
\end{abstract}

Key words. Sun: atmosphere - Sun: filaments - Sun: granulation - Sun: magnetic fields

\section{Introduction}

Filaments (prominences seen on the limb) which are common solar features always occur along lines where the underlying photospheric magnetic field changes sign. They represent regions where magnetic fields are interacting with the plasma in a subtle way in the different parts of the solar atmosphere. The filament's existence in the corona is mainly due to magnetic fields that support dense material against gravity inside dipped arcade loops or flux tubes (Kuperus \& Raadu 1974). The filaments are structures of the solar corona which are anchored at footpoints in the solar photosphere. These footpoints connect with the photosphere with a periodicity of few tens of Mm, which relates to their connection with supergranules (Plocieniak \& Rompolt 1973; Aulanier et al. 1998). Several theorical investigations have proposed models for the formation of filaments based on converging flows produced by turbulence convection in the photosphere (Van Ballegooijen \& Marten 1989; Choe \& Lee 1992; Ridgway \& Priest 1993). Knowledge of photospheric motions over long periods is needed to understand the action of the plasma on the filament. In particular, barbs, footpoints of prominences when observed on the disk, are always associated with parasitic polarities (Martin \& Echols 1994; Aulanier et al. 1998, 1999, 2000; Martens \& Zwaan 2001) implying a reversal of the transverse horizontal magnetic field. The mechanism generating such magnetic configurations must take into account photospheric motions. Martres et al. (1981) found a photospheric velocity field beneath a filament in which lines of zero radial velocity were roughly perpendicular to the direction of the filament. Martin et al. (1985) observed that the formation and evolution of filaments is linked to converging photospheric motions perpendicular to the polarity inversion line (PIL). The successive emergence and cancellation of minor polarities in filament channels have been used to explain the formation and the disappearance of a part of a filament (Schmieder et al. 2006).

In reponse to these observations new theories of filament formation have been developped (Van Ballegooijen 2004; Martens \& Zwaan 2001; Priest 1997; Aulanier et al. 1998; Kuijpers 1997; Ridgway \& Priest 1993). From a theoretical point of view, the field line footpoints are subjected to shearing flows parallel to the PIL (Ridgway \& Priest 1993; Mackay et al. 2000; Mackay \& van Ballegooijen 2006). Some of these models include converging photospheric motions that allow the magnetic configuration to support a dense plasma in the corona.

Most observational studies of filaments have been carried out to describe their properties and structures in the corona and chromosphere. However, the magnetic field contributing to the formation of the filaments can transfer photospheric perturbations into the corona. Very few papers exist on the determination of photospheric motions beneath and in the vicinity of filaments. Such measurements require multi wavelength observations. We use the method described in Roudier et al. (1999) to measure the horizontal flows in the photosphere in the present paper. 


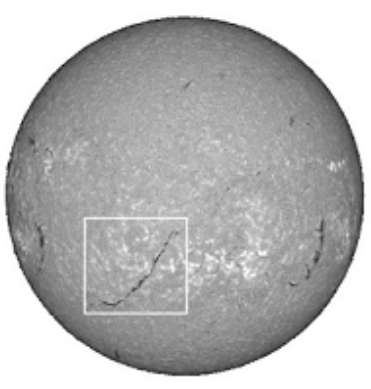

Oct 6, 2004

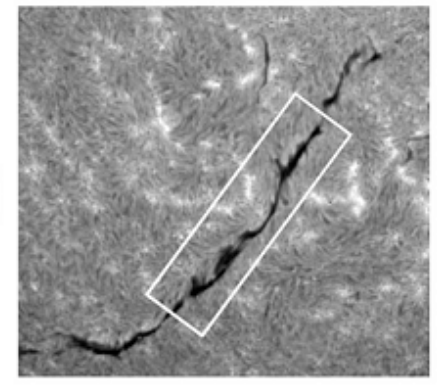

Oct 6, 2004

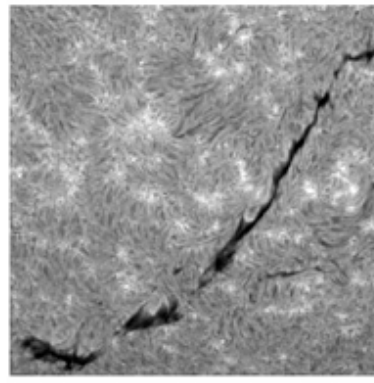

Oct 7, 2004

Fig. 1. (Left) The position of the target filament indicated on an $\mathrm{H} \alpha$ image observed by ISOON on October 62004 and (middle and right) the filament region in the ISOON data on October 6 and 7, 2004 showing how it evolved. The field of view of the DOT filament mosaic shown in Fig. 2 is indicated in the middle image.

The pioneering investigation carried out by Molowny-Horas (1994) on the dynamics of filaments and the underlying photosphere, using photospheric data with moderate resolution, revealed horizontal flows aligned with the direction of the filament and with similar velocity distributions on either side. As distance from the neutral line increases, Molowny-Horas found that parallel flows become less pronounced, and show more of the complex mesogranulation and supergranulation velocity fields. His investigation did not detect any systematic large-scale photospheric motion converging towards the neutral line. The detailed observations of Magara \& Kitai (1999) show evolving, random photospheric motions around the filament channel. They also do not find any convergence toward the filament channel. On the other hand, they observed some unsteady shearing motions around the filament with lifetimes less than $6 \mathrm{~h}$. Recently the OSLO group (Lin et al. 2005) used the Swedish Solar Telescope (SST) to study photospheric flows. They found that flows along network cell boundaries are directed predominantly from one side of the PIL to the other.

Using MSDP data, Malherbe et al. (1983a) found that longlived upward motions often occur in the core of the quiescent filaments. They suggested that such flows $\left(1 \mathrm{~km} \mathrm{~s}^{-1}\right)$ could be related to converging motions in the photosphere and that they are supported by the magnetic field when inverse polarity fields are present (as in the case for most filaments).

In this paper we investigate a filament observed over a large field of view with high spatial resolution. The observations and coalignment between all involved instruments are explained in Sect. 2. The description of horizontal photospheric flows below and in the neighborhood of the filament is illustrated in Sect. 3. The location of the filament relatively to the photospheric network is described in Sect. 4 and formation of parasitic polarities is discussed in Sect. 5. We present in Sect. 6 the photospheric and chromospheric context of the filament eruption on October 7 , 2004. Finally, Sect. 7 contains a summary and discussions of the results.

\section{Observations and data processing}

\subsection{Description of data and instrumentation}

Our data come from a combination of ground and space based telescopes on October 6-8, 2004 during a JOP 178 campaign (http://bass2000 . bagn . obs-mip . fr/jop178/index . html). The target was a quiescent filament seen in Fig. 1 at solar coordinates S16E11 (on Oct. 6). This target was observed by the Dutch Open Telescope (DOT), THEMIS, the Meudon
Solar Tower (MST), The Dunn Solar telescope (DST), the Improved Solar Observing Optical Network (ISOON), TRACE and SOHO/MDI/EIT. All of these instruments tracked this target and observed its eruption on October 7 at approximately 16:30 UT.

Table 1 summarizes the characteristics of all the JOP 178 observations used for our analysis. The ground-based observations are summarized below. The four DOT cameras recorded the same field of view in the G-band, blue continuum, red continuum, Ca II H line center (passband $0.128 \mathrm{~nm}$, Rutten et al. 2004; see Leenaarts et al. 2006, for detail). DOT $\mathrm{H} \alpha$ line center observations on October 6, 2004 at 8:50 UT were used to construct the mosaic in Fig. 2 (Rutten et al. 2004, http://www.dot.iac.es/). THEMIS (http://www.themis.iac.es/) obtained polarimetric and Doppler measurements of this region on October 6-8, 2004. The MSDP spectrograph at MST obtained $\mathrm{H} \alpha$ Doppler velocities of the target on October 6. The DST located at Sacramento Peak (http://www . nso . edu/) obtained high-resolution G-band and $\mathrm{H} \alpha$ time sequences of the target on Oct. 6 and 7, 2004. ISOON, also at Sacramento Peak, (http://nsosp.nso.edu/isoon/), observed the target in $\mathrm{H} \alpha$ full-disk mode on October 6 and 7, 2004.

Observations from space were obtained with TRACE (http://trace.lmsal.com/) and SOHO/MDI (http://sohowww.nascom.nasa.gov/) and are summarized below and in Table 1. TRACE observed the filament region almost continuously on October 6 and 7, 2004. The MDI on board SOHO provided a full-disk longitudinal magnetogram every minute and EIT full-disk observations on October 6 and 7, 2004.

\subsection{Coalignment of the data}

We have coaligned successively all of the data obtained by the different instruments. Our primary goal was to derive the horizontal flow field below and in the vicinity of the filament. Therefore, we had to coalign the white light (WL) TRACE data with the $\mathrm{H} \alpha$ observations from DOT, DST and ISOON. In order to achieve this coalignment, we applied different strategies for each of the ground-based instruments. For the DOT, we first coaligned the CaII H (3968 $\AA$ ) and $1600 \AA$ TRACE observations by using the chromospheric network as a reference. THEMIS and DOT data were coaligned by precisely overlapping the filament visible in $\mathrm{H} \alpha$ in both observations. The time difference between the DOT (8:35) and THEMIS (10:06 to 11:09) 
Table 1. October 6-8 2004.

\begin{tabular}{|c|c|c|c|c|c|}
\hline Telescope & Datatype & Field of view & Pixel size & Cadence & Time UT \\
\hline DOT & $\begin{array}{c}\text { G-band } \\
\text { blue Continuum } \\
\text { red Continuum } \\
\text { CaIIC H line center } \\
\mathrm{H} \alpha \\
\text { (simultaneously) }\end{array}$ & $85^{\prime \prime} \times 323^{\prime \prime}$ & $0.071^{\prime \prime}$ & $60 \mathrm{~s}$ & $8: 50$ October 6 \\
\hline THEMIS & $\begin{array}{l}\text { Magnetic field } \\
\text { Doppler velocity } \\
\text { (simultaneously) } \\
\text { FeI } 6302 \AA \\
\text { H } \alpha\end{array}$ & $120^{\prime \prime} \times 360^{\prime \prime}$ & $\begin{array}{c}0.43^{\prime \prime} \text { along the slit } \\
0.8^{\prime \prime} \text { in the } \\
\text { scanning direction }\end{array}$ & irregular & $\begin{array}{c}10: 06-11: 09 \text { October } 6 \\
9: 10-10: 13 \text { October } 7 \\
10: 44-11: 35 \text { October } 7 \\
14: 30-15: 17 \text { October } 7 \\
8: 30-11: 35 \text { October } 8\end{array}$ \\
\hline MEUDON MSDP & H $\alpha$ Doppler velocity & $240^{\prime \prime} \times 420^{\prime \prime}$ & $0.5^{\prime \prime}$ & $30 \mathrm{~s}$ & $13: 12-13: 42$ October 6 \\
\hline DST & $\begin{array}{c}\text { G-band } \\
\mathrm{H} \alpha\end{array}$ & $83^{\prime \prime} \times 83^{\prime \prime}$ & $\begin{array}{l}0.101^{\prime \prime} \\
0.171^{\prime \prime}\end{array}$ & $20-30 \mathrm{~s}$ & $\begin{array}{l}15: 11-17: 11 \text { October } 6 \\
14: 48-16: 48 \text { October } 7\end{array}$ \\
\hline ISOON & $\mathrm{H} \alpha$ & full-disk & $1.077^{\prime \prime}$ & $1 \mathrm{~min}$ & $\begin{array}{l}14: 05-22: 35 \text { October } 6 \\
13: 37-22: 35 \text { October } 7\end{array}$ \\
\hline TRACE & $\begin{array}{c}\text { white Light } \\
\text { Continuum } 1600 \AA \\
\text { Fe IX-X } 171 \AA \\
\text { Fe XII } 195 \AA \\
\text { (not simultaneously) }\end{array}$ & $384^{\prime \prime} \times 384^{\prime \prime}$ & $0.5^{\prime \prime}$ & $30-180 \mathrm{~s}$ & $\begin{array}{l}\text { continuous October } 6 \\
\text { continuous October } 7\end{array}$ \\
\hline $\mathrm{MDI} / \mathrm{SOHO}$ & $\begin{array}{c}\text { magnetogram } \\
\text { Doppler velocity }\end{array}$ & full-disk & $1.96^{\prime \prime}$ & $1 \mathrm{~min}$ & $\begin{array}{c}20: 49-23: 49 \text { October } 6 \\
9: 44-22: 50 \text { October } 7\end{array}$ \\
\hline EIT/SOHO & $\begin{array}{c}\text { NiI } 6768 \AA \\
\text { He II } 304 \AA \\
\text { Fe IX-X } 171 \AA \\
\text { Fe XII } 195 \AA\end{array}$ & full-disk & $2.63^{\prime \prime}$ & $\begin{array}{l}96 \text { min continuous } \\
\qquad 4 \mathrm{fr} / \mathrm{h}\end{array}$ & $\begin{array}{c}\text { October } 6 \text { and } 7 \\
\text { continuous October } 6 \\
\text { continuous October } 7\end{array}$ \\
\hline
\end{tabular}

observations is small enough that the filament maintains its shape sufficiently to allow coalignment of these data at the $\pm 1^{\prime \prime}$ level. To control the quality of this coalignment, we also compared the bright regions in the CaII $\mathrm{H}$ and in the G-band, with the magnetic maps from THEMIS. To use TRACE WL data to measure horizontal flow with respect to the filament on ISOON observations, the alignment was performed in two steps: first we coaligned the DST G-band and $1600 \AA$ TRACE data; second, we coaligned the $\mathrm{H} \alpha$ filament and the bright features visible simultaneously on DST and ISOON observations. This enabled us to coalign TRACE and ISOON data at $\pm 1^{\prime \prime}$.

The co-alignment between SOHO/MDI magnetograms and ISOON $\mathrm{H} \alpha$ images was performed by adjusting the chromospheric network visible in $\mathrm{H} \alpha$ (ISOON) and the amplitude of longitudinal magnetogram of MDI with an accuracy of one pixel $\left(1.96^{\prime \prime}\right)$.

\subsection{Filtering and velocity field}

We have extracted time sequences of TRACE WL data to correspond with the respective $\mathrm{H} \alpha$ observations. The subsets of the TRACE WL sequence used to obtain the horizontal velocities are respectively: from 7:45 to 10:06 UT on October 6, 2004 corresponding to the DOT observations, from 14:48 to 17:31 UT on October 6, 2004 corresponding to ISOON observations and from 13:30 to 18:05 on October 7, 2004 also corresponding to ISOON observations. The TRACE WL images were rigidly-aligned and filtered for p-modes in $k-\omega$ space (threshold phase velocity of $4 \mathrm{~km} \mathrm{~s}^{-1}$ ) to remove five-minute oscillations. Next, horizontal flow vectors were derived using local correlation tracking (LCT, November \& Simon 1988). The flow velocities were derived using a correlation window of $3^{\prime \prime} \times 3^{\prime \prime}$ corresponding to a scale that can faithfully track granules (Rieutord et al. 2001). As the (measured) velocity field is purely two-dimensional, two quantities are relevant to describe flow structures: these are the divergence $D=\partial_{x} v_{x}+\partial_{y} v_{y}$ and the $z$-component of the vorticity $\zeta=\partial_{x} v_{y}-\partial_{y} v_{x}$. The MDI data were also rigidly aligned and filtered for $\mathrm{p}$-modes in $k-\omega$ space in the same manner used for the TRACE WL data.

\section{The photospheric flow pattern below and in the neighborhood of the filament}

Figure 2 shows a mosaic at 8:50 UT of the $\mathrm{H} \alpha$ filament structure that was observed in the southern solar hemisphere (S16E11) on October 6, 2004 during the decaying phase of the solar cycle. This filament has a sinistral chirality which is a normal configuration in that hemisphere. The smallest chromospheric features on the image show that the angular resolution is around $0.5^{\prime \prime}$ over the field of view of $95^{\prime \prime} \times 323^{\prime \prime}$. The $5^{\prime \prime}$ radius circles indicate the region of apparent footpoints.

As a way to visualize the horizontal flow, we introduce passive scalars (corks), and allow them to move with the local horizontal velocity over extended time periods. The corks eventually arrange themselves into a network at supergranular scale. Figure 3 shows the cork trajectories resulting from the measured horizontal velocities over a period of $5 \mathrm{~h}$ superimposed on the location of the filament. The distribution of horizontal velocity amplitude lies between 0 and $1.2 \mathrm{~km} \mathrm{~s}^{-1}$ and peaks at $0.3 \mathrm{~km} \mathrm{~s}^{-1}$. We observe that the corks are expulsed from diverging cells with dimensions corresponding from meso- to super-granular scales as expected. Some of the cork trajectories cross the filament at various locations along its length. The divergence map (Fig. 3) does not exhibit any peculiar behaviour below the filament and indicates that the horizontal flow is similar over the entire field of view. 


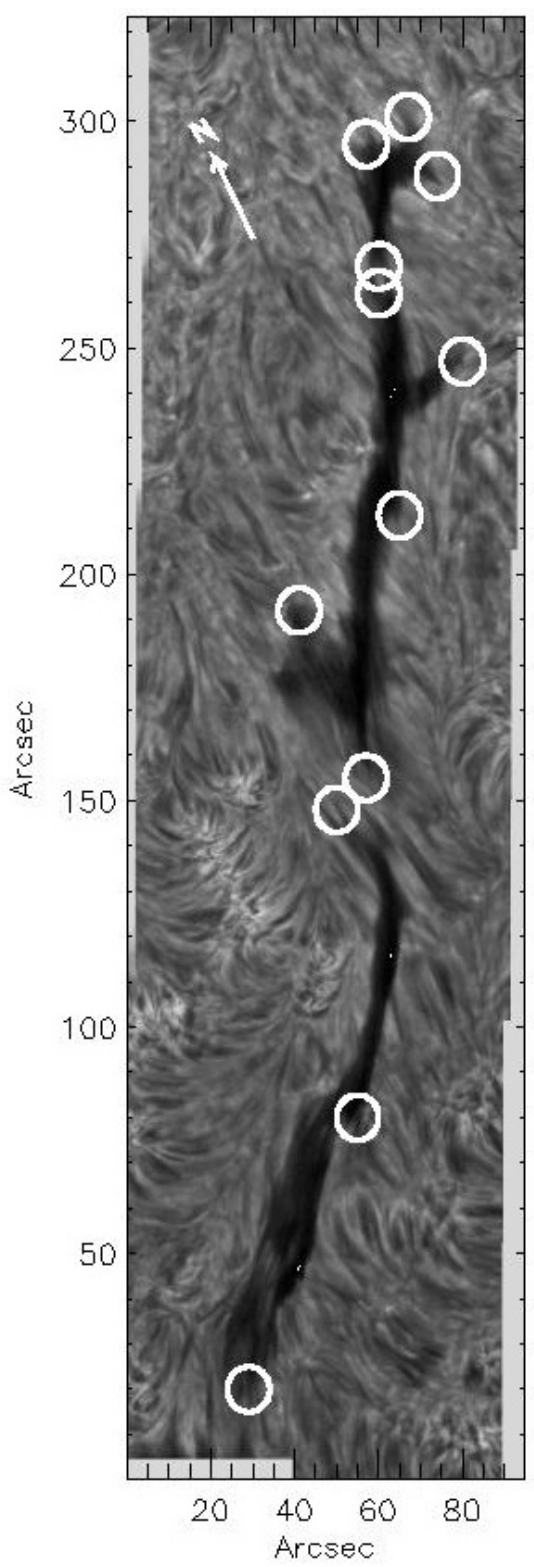

Fig. 2. A mosaic of the filament in $\mathrm{H} \alpha$ observed with the DOT, made from 6 frames selected at about 8:50 UT on October 6, 2004. The circles indicate the footpoint locations of the filament. The arrow indicates solar north.

Figure 4 shows the location of the filament observed in $\mathrm{H} \alpha$ by ISOON later in the afternoon on October 6, 2004, superposed on the divergence field derived from the horizontal velocities measured on TRACE WL data. As was the case with the DOT data taken earlier on October 6, we do not observe peculiar photospheric motions below or in the neighborhood of the filament. We observe the well-known pattern of meso- and super-granular cells everywhere. In particular, neither set of observations reveal the predicted large-scale converging flows often invoked in filament formation. Using larger temporal windows for avering the horizontal flow window or using larger spatial windows to compute the velocity field with the LCT algorithm did not change the result. One explanation could be that we observed a mature filament that was not in its early formation phase. However, at a few locations along the filament, we observe areas of strong divergence, three of which particularly stand out in the top part of the filament. For example the
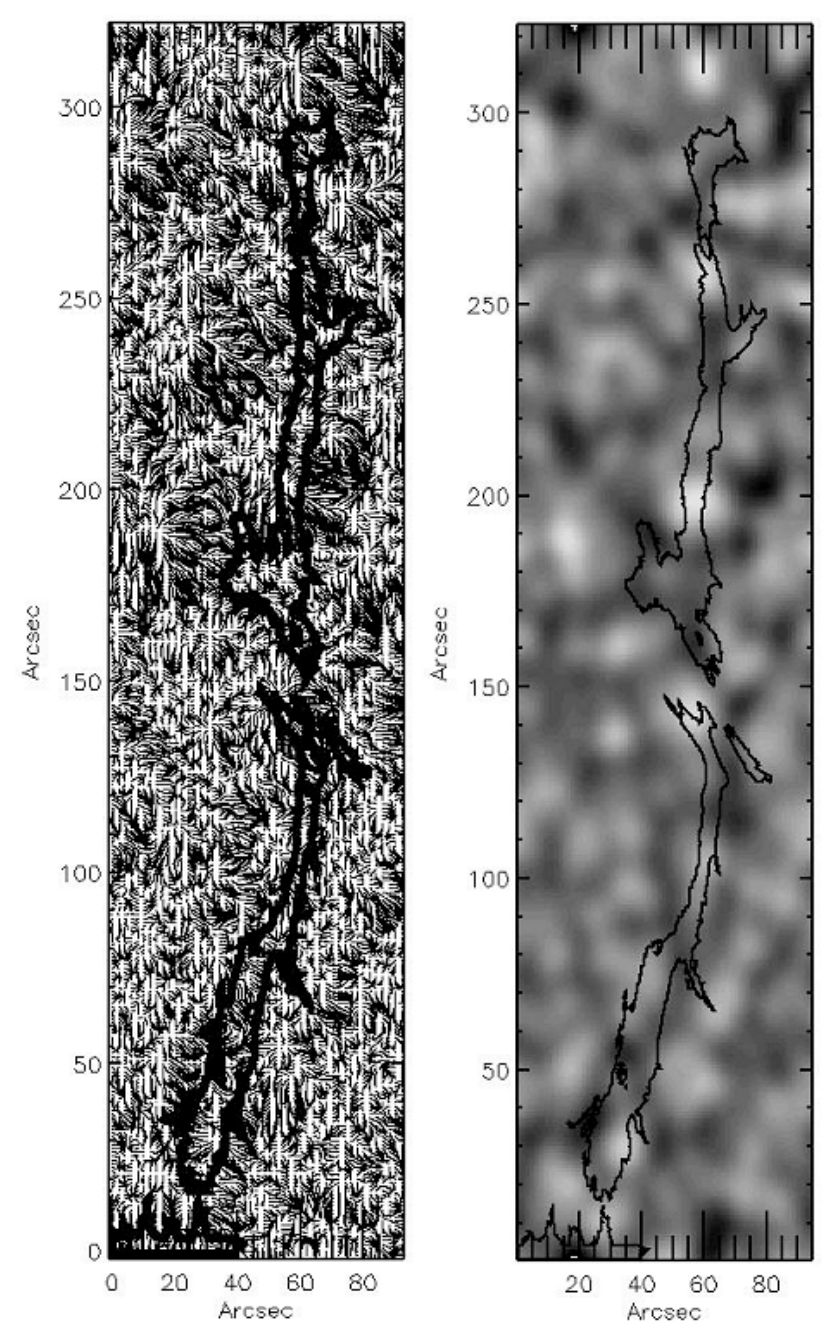

Fig. 3. (Left) cork trajectories (passive scalars) computed from horizontal velocities obtained by tracking the TRACE WL data over a $5 \mathrm{~h}$ period are superimposed on the location of the filament (thickline, isocontour in the $\mathrm{H} \alpha$ intensity map) observed with the DOT. On the right, divergence map computed from the horizontal photospheric velocities (TRACE data) superimposed on the location of the filament observed with the DOT. Bright are diverging and dark converging flow.

structure at the coordinates $(55,149)$ in Fig. 3 (DOT data) remains visible in Fig. 4 at about 15-16 UT, i.e. 6 h later at the coordinates $(210,170)$ and also in the DST data (not shown). The divergence at $(55,149)$ is located in a gap of the filament which separates two main footpoints. The divergence at $(58,259)$ in the DOT data is located where the filament breaks up later in the afternoon, as seen in the ISOON data. This could indicate that divergent flow contributes to the creation and maintenance of the gap in the filament.

Finally, Fig. 5 shows the vorticity of the horizontal flow relatively to the filament. We note that in our observations the footpoints of the filament are generally located in areas of positive vorticity. However, we also see a strong negative vorticity in the lower part of the filament at the location $(40,60)$.

\section{Filament and network, and chromospheric dynamic of the filament}

In this section, we compare the network obtained by tracking passive corks in the measured photospheric flow field with 


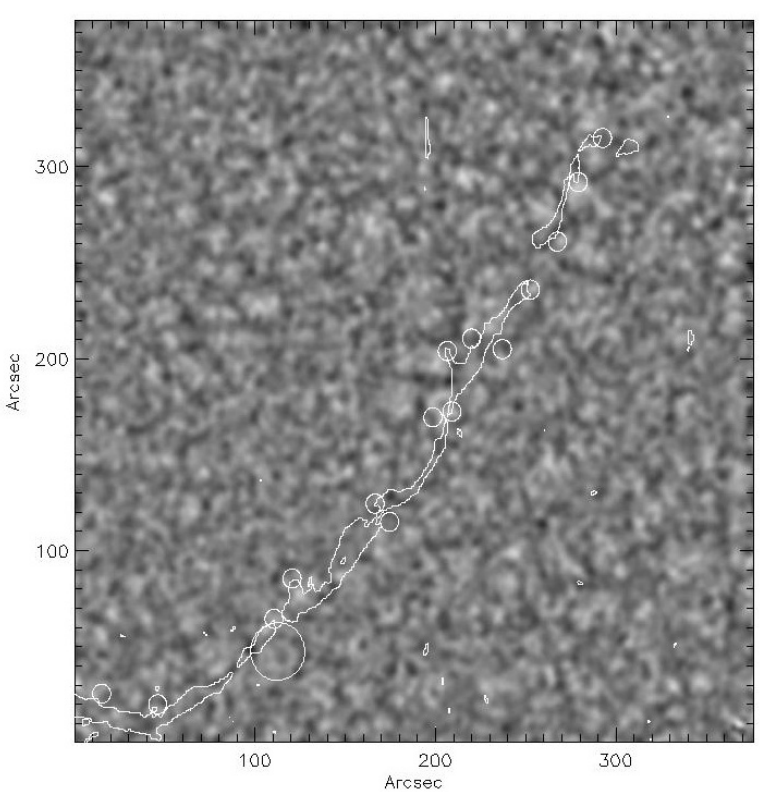

Fig. 4. A divergence map computed from the horizontal photospheric velocities on October 6, 2004 between 14:48 and 17:31 UT, using the TRACE WL data the location of the filament seen in $\mathrm{H} \alpha$ by ISOON superimposed. Bright areas are diverging and dark areas are the converging flow. Solar north is located at the top of the figure. The large white circle indicates the location of the divergence which is still visible on Oct. 7 2004, while the smaller circles show the filament footpoints.

properties of the chromosphere around the filament. Following Lin et al. (2005), we assume that sub-structure seen in $\mathrm{H} \alpha$ at semi-regular spacing pointing away from either side of the main filament body, the so-called barbs, represent magnetic footpoints that connect into the photosphere. Our objective in this section is two fold: to verify the quality of the coalignment between the various data sets, and to compare the location of the barbs with the network boundaries, irrespective of polarity considerations. We then describe the evolution of the chromospheric properties of the filament with respect to the filament footpoints in the photosphere. The following section discusses the magnetic field associated with the filament.

Figure 6 shows the final location of the corks after propagating for 5-h in the flow field (computed from TRACE data), superimposed on a CaII map. In this figure, we verify the colocation of the network formed by the corks with the bright CaII features representing the chromospheric network. The small differences between the cork positions and bright $\mathrm{CaII}$ features is partly due to the short interval $(5 \mathrm{~h})$ over which the corks were allowed to propagate compared to the much longer lifetime of the chromospheric network. The good correspondence between the corks and the CaII network confirms the alignment between the observations from the different ground and spacebased instruments.

Similarly, Fig. 7 shows the final location of the corks superimposed on the $\mathrm{H} \alpha$ filament as seen by ISOON later on October 6, 2004. The figure shows a rather good correlation between cork location and the magnetic network visible as bright features at superganular boundaries.

At the photospheric level, the solar granulation does not exhibit any special behaviour below or near the filament. However, several supergranulation cells are seen to cross the PIL as observed by Lin et al. (2005). The $5^{\prime \prime}$ radius circles in Fig. 6 indicate the locations of apparent end points of the filament barbs. We find that $75 \%$ of the observed barb end points fall within the

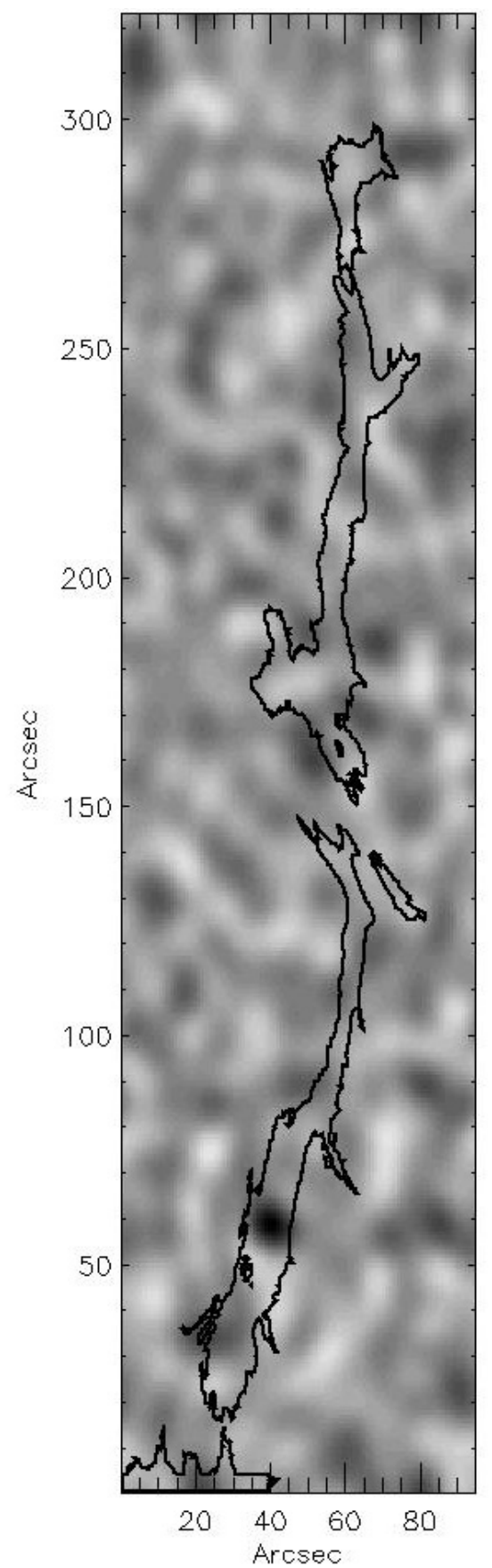

Fig. 5. A vorticity map computed from the horizontal photospheric velocities (TRACE data) with the location of the filament observed with DOT superimposed.

network boundaries (defined both by corks and CaII), which is in good agreement the earlier findings of Lin et al. (2005).

Figure 8 overlays $\mathrm{H} \alpha$ intensity isolines showing the location of the filament on chromospheric Doppler velocities observed in $\mathrm{H} \alpha$ at Meudon (13:36 UT). We observe a classical blueshift in the filament core indicating that the material is upflowing (between 0.5 and $2 \mathrm{~km} \mathrm{~s}^{-1}$ ). We observe a strong upflow $\left(4 \mathrm{~km} \mathrm{~s}^{-1}\right)$ at the location of the gap in the filament $(210$, $90)$ in Fig. 8. However, the dynamics in the filament footpoints are more complex with mixed, rapidly evolving, upflows and downflows (lying between -2 to $2 \mathrm{~km} \mathrm{~s}^{-1}$ ). This is confirmed by Doppler velocities (Fig. 9) measured in $\mathrm{H} \alpha$ by THEMIS (10:06-11:09 UT) a few hours before the Meudon observation. 


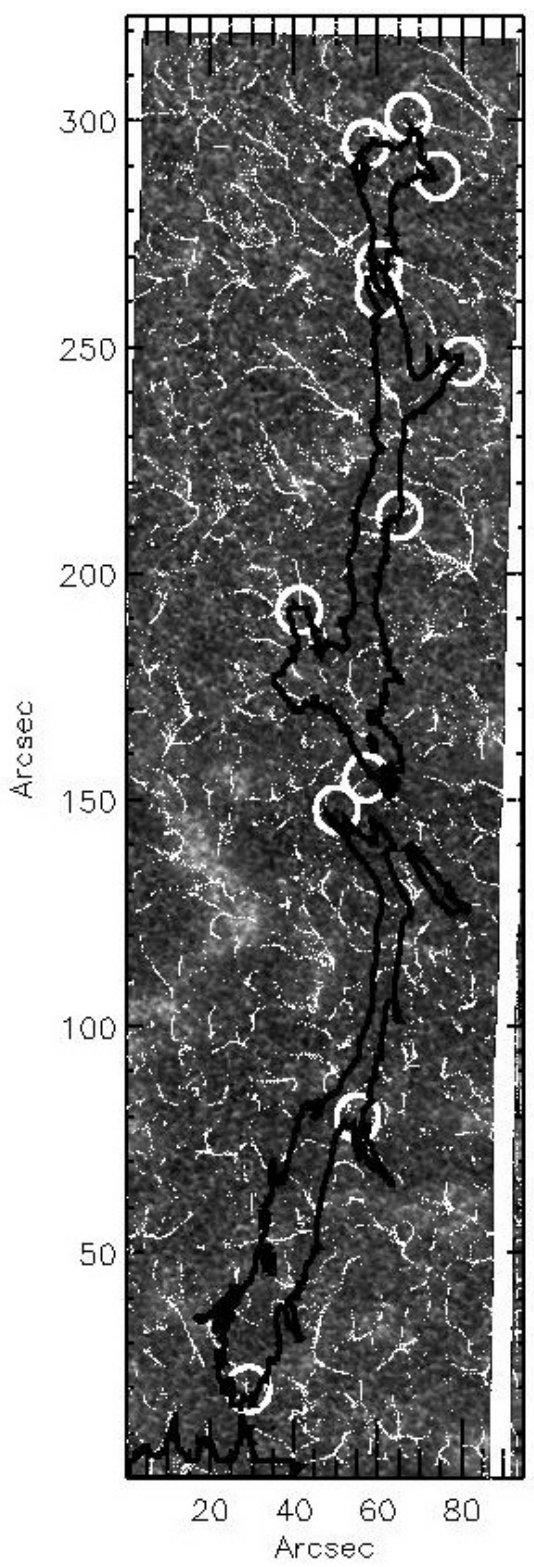

Fig. 6. A chromospheric image taken with the DOT in $\mathrm{Ca}$ II $\mathrm{H}$ (3968 $\AA$ ), showing the atmosphere about $500 \mathrm{~km}$ above the photosphere. Superimposed are the final corks positions after 5-h of evolution of the flow field and an isophote showing the location of the filament in $\mathrm{H} \alpha$ with circles indicating the footpoints. The angular resolution is close to $0.2^{\prime \prime}$ over the whole field.

In this earlier THEMIS data, we observe a general upflow at the footpoints of the filament. Both upward and downward flows are visible at the top of the filament position $(64,285)$ in Fig. 9, while no such motions are observed a few hours later in the Meudon data at the same location. Our observation for the upflowing material in the filament core and the complex dynamics associated with filament footpoint, confirm the previous results of Malherbe et al. (1983b), Schmieder et al. (1984) and Schmieder et al. (1991).

\section{Parasitic polarities}

We have directly measured the magnetic field with the THEMIS/MTR polarimeter (Fig. 9). A possible link between horizontal photospheric flows and the location of parasitic

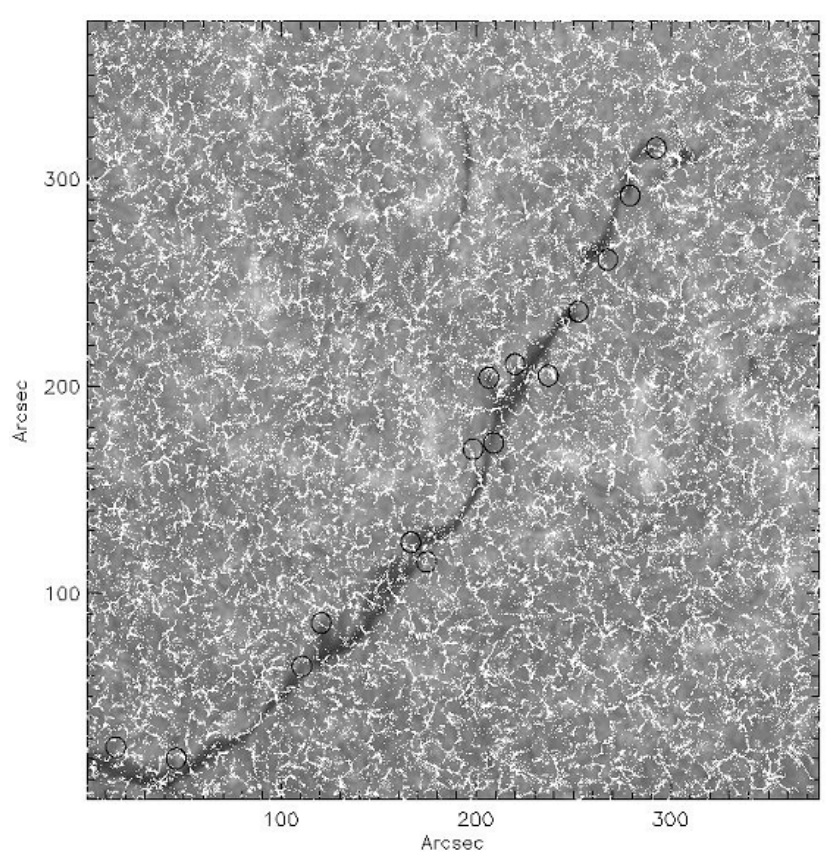

Fig. 7. The filament region observed in $\mathrm{H} \alpha$ by ISOON on October 6 , 2004, between 14:48 and 17:31 UT. The final positions of the corks computed from 5-h of evolution of the flow field, are superimposed. Solar north is located at the top of the figure.

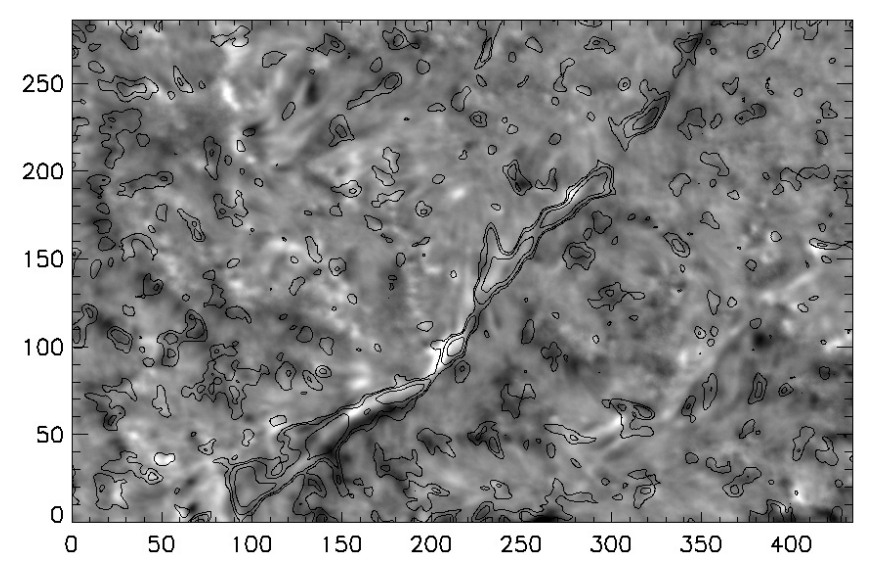

Fig. 8. $\mathrm{H} \alpha$ MSDP spectrograph observation from Meudon on Oct. 6, 2004 at 13:36 UT. Dark represents downflow and bright upflow. Solar north is located at the top of the figure.

polarities was investigated using the THEMIS magnetic map and the horizontal flows computed with the TRACE WL data.

THEMIS measured the magnetic field in the filament region in FeI $6302 \AA$ A between 10:06 and 11:09 UT on October 6. The amplitude of the longitudinal magnetic field shown in Fig. 9 lies between \pm 447 Gauss, with the highest absolute values being saturated on this map. The cork trajectories computed over a 5-h period and the filament as seen in $\mathrm{H} \alpha$ are superimposed on the magnetic map. We observe that the filament lies above the polarity inversion lines (PILs) seen within the filament channel. We also note the presence of parasitic polarities on both sides of the PIL as expected. For example in Fig. 9 we find strong positive parasitic polarities (shown in red) located at the coordinates $(70$, $248),(70,190),(80,250),(85,130)$ and strong negative parasitic polarities (shown in green) at $(20,25),(48,125)$.

We now examine the interaction between these parasitic polarities and the flows and in particular with the supergranules 

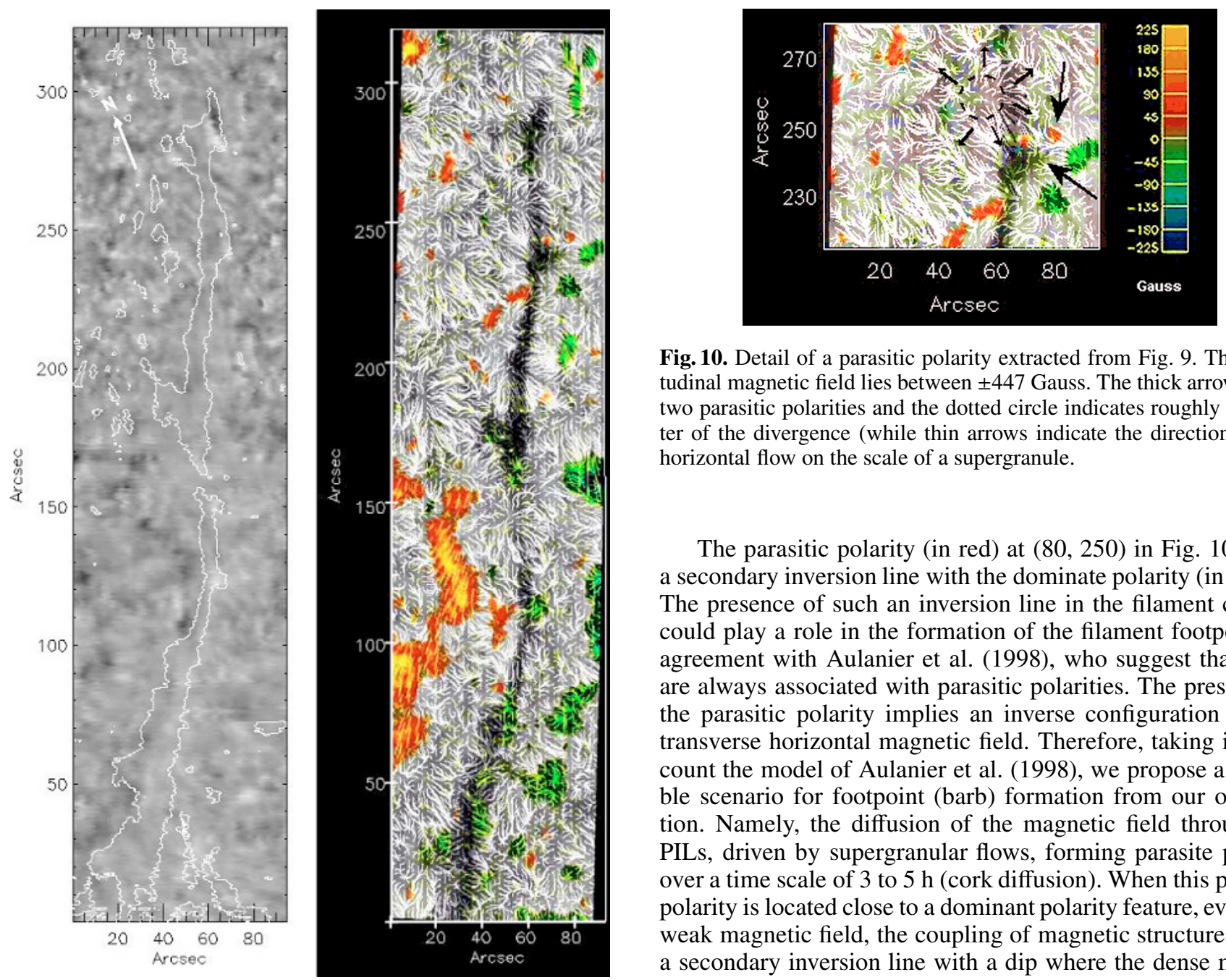

Fig. 10. Detail of a parasitic polarity extracted from Fig. 9. The longitudinal magnetic field lies between \pm 447 Gauss. The thick arrows show two parasitic polarities and the dotted circle indicates roughly the center of the divergence (while thin arrows indicate the direction) of the horizontal flow on the scale of a supergranule.

The parasitic polarity (in red) at $(80,250)$ in Fig. 10 forms a secondary inversion line with the dominate polarity (in green). The presence of such an inversion line in the filament channel could play a role in the formation of the filament footpoints in agreement with Aulanier et al. (1998), who suggest that barbs are always associated with parasitic polarities. The presence of the parasitic polarity implies an inverse configuration for the transverse horizontal magnetic field. Therefore, taking into account the model of Aulanier et al. (1998), we propose a plausible scenario for footpoint (barb) formation from our observation. Namely, the diffusion of the magnetic field through the PILs, driven by supergranular flows, forming parasite polarity over a time scale of 3 to $5 \mathrm{~h}$ (cork diffusion). When this parasitic polarity is located close to a dominant polarity feature, even with weak magnetic field, the coupling of magnetic structures forms a secondary inversion line with a dip where the dense material can be retained against gravity, on the side of the main prominence body. This agrees with the previous works of Aulanier \& Schmieder (2002), López Ariste et al. (2006) and Schmieder et al. (2006) who show the importance of small polarities in the formation of dips.

However, since we have not observed the temporal evolution of the magnetic field, we cannot exclude the possibility that the parasitic polarities emerged locally and that they are not transported by the photospheric flows. Aulanier (1998) found that the angle barbs formed with the filament correlated with the motion of the parasitic polarity involved in its formation. This implies a relationship between polarity changes and photospheric motions. In Fig. 9 several supergranulation cells are seen to cross the PIL. We find several examples of parasitic polarities linked to divergent flow such as those in Fig. 9 at $(48,125)$ (green) or $(70,190)$ red, low amplitude). Three other examples appear to be related to the evolution of the convective flow: $(43,255)$ (green), $(85,130)$ (red) and, $(20,25)$ (green). In general the parasitic polarities are located close to maxima in the amplitude of the horizontal velocity.

We have also computed the photospheric horizontal velocities, on October 7, 2004, using the TRACE data between 9:40 and 11:00 UT. Figure 11 shows a THEMIS magnetogram (between 9:10 and 10:13 UT) and the final corks locations after 5-h. We note a very good correspondence between the position of the corks and the magnetic concentrations at the boundaries of the supergranules, indicating a good alignment between the different data as in Sect. 4. We observe from the cork trajectories (not shown) that corks are also transported through the filament 


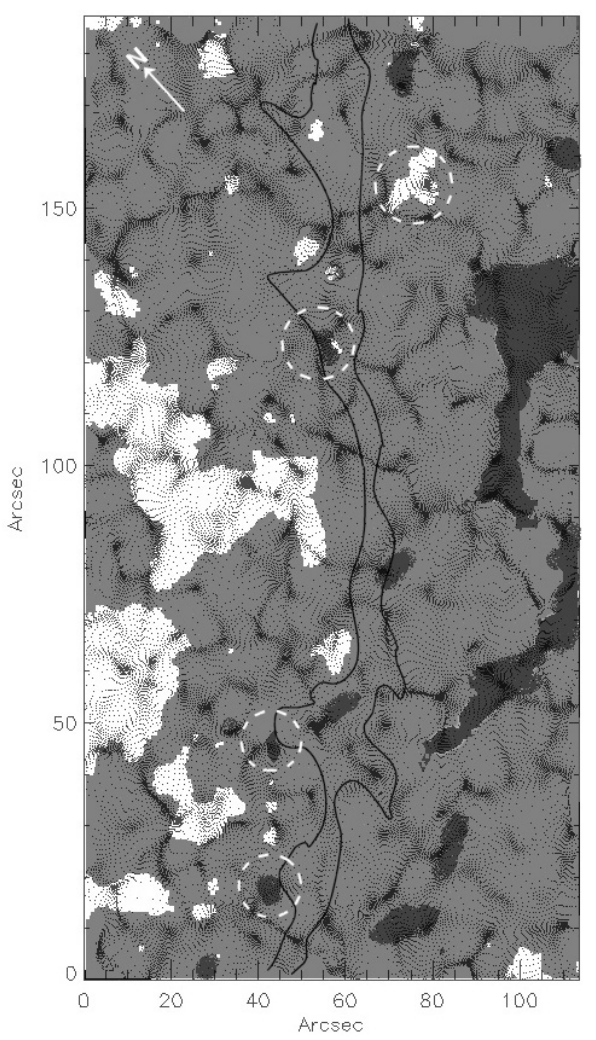

Fig. 11. A THEMIS magnetogram taken on October 7, 2004, between 9:10 and 10:13 UT, with cork final positions superimposed. Some parasitic polarities are indicated by dotted circles. The arrow indicates solar north.

channel. The final position of some corks correspond to parasitic polarities (circles in Fig. 11), which appear to have been transported to the other side of the neutral line by the horizontal flows. Three of them, the lowest ones in Fig. 11 are located close to footpoints of the filament. This observation agrees well with the results obtained on the previous day. However, the pair of polarities described in Fig. 10 (Oct. 6) is no longer visible on the Oct. 7 THEMIS magnetogram, due to the evolution of the magnetic field.

\section{Filament eruption}

The filament erupted on October 7, 2004 in the manner most often observed. The filament completely disappears over about $3 \mathrm{~h}$ between 16:30 and 19:40 UT. As described by Martin et al. (1985), at the start of the eruption, the centre of the filament first expands outward with the far ends remaining attached at their photospheric footings. Next it developed the classic arch form as it disappeared from view against the solar disc. The eruption of a filament often produces a coronal mass ejection (CME) and sometimes a large two-ribbon flare. In this case the filament erupted around 16:30 on October 7, 2004, and a CME was observed at around 19:00 UT with the LASCO-2/SOHO coranagraph and a two ribbon flare was observed with the SOHO/EIT. In this section we investigate the flows associated with this eruption.

\subsection{Parasitic polarities and brightness enhancements}

Before the filament erupts, we observe an increase in brightness at three separate locations in the ISOON $\mathrm{H} \alpha$ data. These
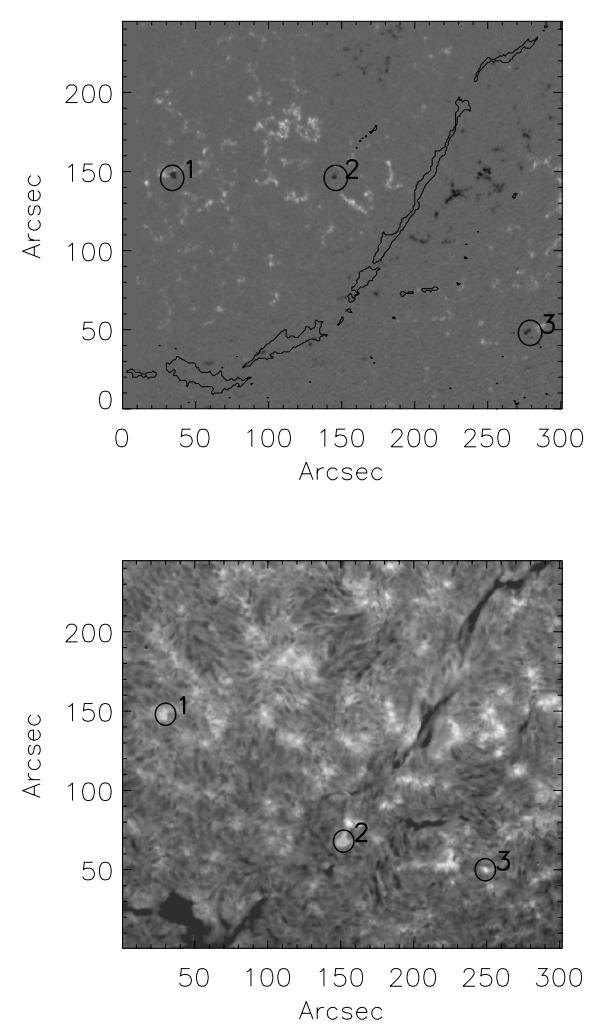

Fig. 12. Top: A MDI magnetogram observed on October 7, 2004 at 19:14 on which the three parasitic polarities are visible. Bottom: An ISOON H $\alpha$ map on October 7, 2004 at 16:30 UT. Solar north is directed toward the top of the figure.

three brightenings, visible in Fig. 12 (bottom) start to increase in brightness respectively at 17:04 (\#1), 17:15 (\#2) and 17:27 (\#3). Only Feature \#1 is near a parasitic polarity observed in the magnetogram. Features \#1 and \#2 have negative polarity while feature \#3 is positive.

SOHO/MDI acquired magnetograms continuously throughout the filament eruption. No changes in the longitudinal magnetic field were detected during the eruption. Only three features with strong parasitic polarity (PP), relatively to the local background magnetic field, appeared before and after the eruption. The PP \#1 in Fig. 12 (top) appears at 13:04 UT with the emergence of new field at around 16:30, which corresponds to a brightness increase in the ISOON $\mathrm{H} \alpha$ observation around 17 UT. PP \#2 appears at about 18:29, after the eruption. Finally, PP \#3 emerges before the eruption at 12:54 and appears to split at 19:04, after the eruption. PP numbers \#1 and \#3, which appear before the eruption, are located at distances of 137" and 123" from the filament, respectively. Both lie outside the TRACE $171 \AA$ field of view, thus we cannot determine if a link exists between these features and the filament. Unfortunately TRACE did not observed in $171 \AA$ between 16 and 18 UT, during the impulsive phase of the filament eruption. At 15:30 UT, before the eruption, only faint minor loops have formed over the filaments body, as seen in $171 \AA$. At 18:32 UT, the middle sections of the loops have brightened considerably and some appear to touch down at a newly created brightening located inside the filament while others continue to loop over the entire filament. From 18:32 to 23:12 UT the newly created brightening continues to brighten and grow in size while propagating down the length of the filament. 


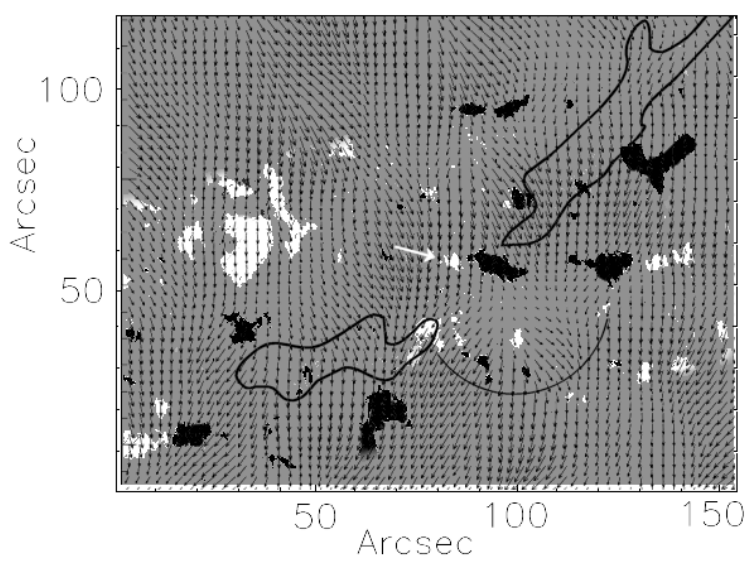

Fig. 13. Magnetic field measured by THEMIS on October 7, 2004 between 14:30-15:17 UT, with the SE extremity of the contour of the filament, and with horizontal photospheric flows, measured with TRACE data, superimposed. The arrow indicates a magnetic feature which is observed to move and interfer with the close opposite polarity (on its right) in the MDI magnetograms (see text). The highest absolute values beeing saturated on this map. The location of the persistent velocity divergence is shown by the arch of the circle. North is located at the top of the figure.

The EIT observations in $284 \AA$, $195 \AA$ and $171 \AA$ taken before (at about 13 UT) and after (at about 19 UT) the filament eruption show a clear reorganization of the magnetic field in the corona and the classic two-ribbon flare configuration.

\subsection{Photospheric flows during the eruption}

To investigate the photospheric motions below and near the filament eruption, we used MDI Doppler data from 9:44 to 22:50 UT and the one-minute cadence TRACE WL observations. The time sequence of Doppler velocities shows only the expected slow evolution of supergranules throughout the entire field-of-view. There were no peculiar changes in the pattern associated with the eruption. To detect the presence of persistent large-scale horizontal flows, we applied LCT with a correlation window of $8^{\prime \prime} \times 8^{\prime \prime}$ to $13 \mathrm{~h}$ of the MDI Doppler data (between 9:50 and 22:50). The resulting horizontal velocities do not show any large-scale organization.

Following the same procedure used for the October 6, 2004 data, we coaligned the October 7, 2004 ISOON H $\alpha$ and TRACE WL data in order to measure the horizontal velocities below and near the filament during its eruption. The horizontal flow vectors were derived by applying LCT with a correlation window of $3^{\prime \prime} \times 3^{\prime \prime}$. A detailed inspection of the velocity vectors and their divergence and vorticity fields did not reveal any peculiar evolution or change of the horizontal flow before or during the filament eruption.

However, at the location where the filament starts to disappear, we observe a persistent divergence of the flow visible before and after the filament eruption. This divergent flow was also present on October 6, 2004, implying a strong continuous flow that remains at the same location over an extended time period.

Figure 13 shows the horizontal velocity measured with TRACE data superimposed on the line-of-sight (LOS) magnetic field measured by THEMIS on October 7, 2004 between 14:30 and 15:17 UT. We observe that parasitic and normal polarities are probably swept by the continuous diverging horizontal flow which is also where the filament gap appears at the beginning of the eruption. Such purely horizontal motions could destabilize

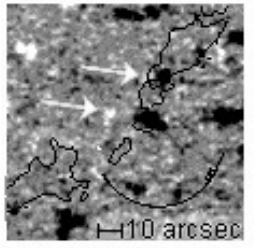

$11: 43$

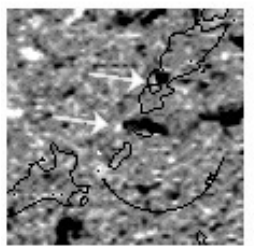

$15: 40$

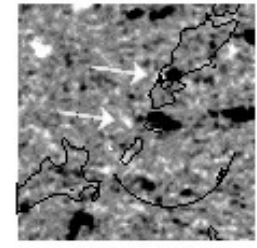

$13: 45$

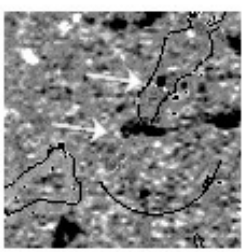

$16: 02$

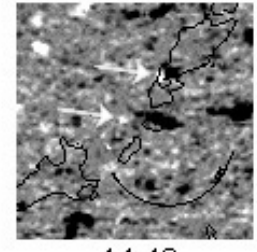

$14: 40$

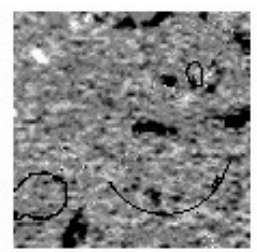

$16: 40$
Fig. 14. A time sequence of the longitudinal magnetic field measured by MDI on October 7, 2004 between 11:43-16:40 UT, with the SE extremity of The filament superimposed, as in Fig. 13. The arrows indicate the structures which move with the photospheric flows towards regions of opposite polarity. The location of the persistent velocity divergence is shown by the arch of the circle. Solar north is located at the top of the figure.

the filament and lead to the sudden filament disappearance (Lin et al. 2001).

Inspection of the MDI magnetograms permits us to study the evolution of the stronger magnetic features seen in the THEMIS data, as was done in Schmieder et al. (2006). Figure 14 shows the time evolution of the MDI magnetograms between 11:43-16:40 UT, in the SE extremity where the eruption begins. We observed two magnetic structures, indicated by arrows on Fig. 14, that move with the photospheric flow towards regions of opposite polarities. The upper structure disappears between 16:05 and 16:10 UT and the lower one between 16:20 and 16:30 UT when they interact with the opposite polarities. These phenomena could initiate the reorganization of the magnetic field and could be candidates to start the destabilization of the filament, which begins erupting at about 16:30 UT.

However, from our observations, we cannot exclude the possibility that the eruption of the filament could be related to physical conditions in the upper atmosphere (chromosphere or corona) rather than the effects of the photospheric motions acting on the magnetic structures.

Finally, from the THEMIS vector magnetic field measurement, we observe a modification of the orientation of the transverse magnetic field before and after the filament eruption. The orientation of the transverse magnetic field in the network has changed between the magnetograms obtained on the 6-8 October, 2004. The azimuth is defined as the angle between the transverse component of the magnetic field and solar north. Figure 15 displays azimuth histograms of the network magnetic field obtained on October 6-8. These histograms have well defined peaks, indicating a strong homogeneity of the azimuth of the network magnetic field. The mean values are $155^{\circ}$ (on October 6), $180^{\circ}$ (or $0^{\circ}$ ) (on October 7) and $45^{\circ}$ (on October 8 ). This change may relate to the activity of the filament which disappears on 7 October and reforms the following day. Projection effects, which change with solar rotation, are negligible relatively to the amplitude of the observed azimuth variation. 

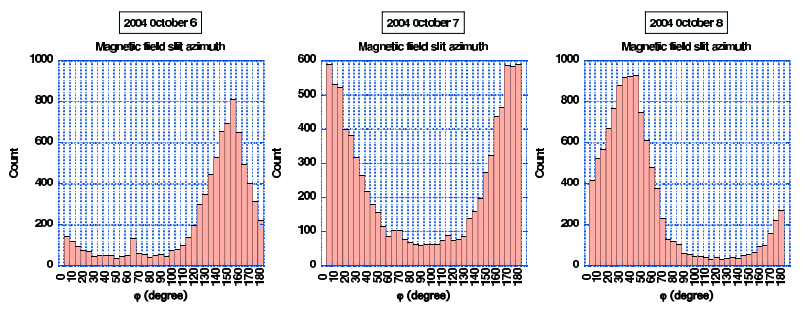

Fig. 15. Azimuth histograms of the network magnetic field obtained for the value of $\alpha \mathrm{B}>45$ Gauss, where $\alpha$ is the magnetic filling factor, on October 6-8.

\section{Discussion and conclusion}

We have presented multi-wavelength observation of the evolution and eruption of a filament obtained during the JOP 178 campaign on October 6 through 8, 2004. We analyzed the photospheric motions below and in the immediate neighborhood of the filament to search for systematic flows that could both sustain the filament and lead to its eruption. We did not observe any large-scale flows converging toward the filament channel that might have contributed to its formation in agreement with the results of Magara \& Kitai (1999). We found that the photospheric motions in the vicinity of the filament exhibit the same properties as those in the remainder of our field-of-view which contains mainly quiet sun. Perhaps motions converging toward the filament exist in the early phases of its formation, but neither our observation nor those of Magara \& Kitai (1999) observe this phase. We observe that several supergranulation cells cross the Polarity Inversion Line. Our observations show that the supergranules can play a role in the transport of parasitic polarities through the filament channel and thus contribute to the formation of barbs (filament footpoints). We presented a detailed example in Sect. 5 where one clearly sees the formation of a secondary magnetic dip at the location of a footpoint.

We also investigate the evolution and eruption of the filament. Regarding the evolution of the filament, On October 7, 2004, we observed an eruption of the filament at approximately 16:30 UT which was followed by a CME visible with the LASCO2/SOHO coronagraph at about 19:00 UT. Before and during the eruption, we detected only a few changes in the MDI/SOHO magnetograms. The horizontal photospheric velocities are similar over the entire field of view and show only the classic evolution of the meso- and super-granulation. However, we observed two phenomena which could cause the filament eruption. The first is the transport of parasitic and normal polarities by a continuous diverging horizontal flow, lasting at least one day, located in the filament gap where the filament starts to disappear. Such a purely horizontal motion could lead to the destabilization of the filament and to the sudden filament disappearance (Lin et al. 2001). The second is the mixing of opposite polarities induced by the horizontal flows which in turn implies a reorganization of the magnetic field at the origin of the filament eruption.

However, since we do not have high spatial and temporal resolution measurements of the magnetic field needed to continuously observe its evolution, we cannot exclude the possibility that the eruption is triggered by physical conditions in the upper atmosphere (chromosphere or corona).

One very interesting observational result was the reorientation by $70^{\circ}$ (or $110^{\circ}$ ) of the transverse field after the eruption seen in the daily vector magnetograms obtained with
THEMIS. Further observations of field changes are required to understand if such shifts in the direction of the field are commonly associated with filament eruptions, and if so, what causes them.

To further elucidate the contribution of photospheric motions to the formation, evolution and eruption of a filament, multiwavelength observations over extended time periods, with both high temporal and spatial resolution, especially for the magnetic field, are needed. The HINODE (Solar B) satellite is equipped to provide such observations.

Acknowledgements. This work was supported by the Centre National de la Recherche Scientifique (C.N.R.S., UMR 5572 and UMR 8109), by the Programme National Soleil Terre (P.N.S.T.) and European OPTICON transnational Access Programme. SOHO is a mission of international cooperation between the European Space Agency (ESA) and NASA. This work was supported by the European commission through the RTN programme (HPRN-CT-2002-00313). We wish to thank THEMIS, DOT, DST, ISOON, SOHO/MDI, SOHO/EIT, TRACE Teams and Ch. Coutard for their technical help. We are indebted to G. Aulanier, P. Mein and N. Mein for many discussions.

\section{References}

Aulanier, G. 1998, Thesis Université Paris VI Pierre et Marie Curie

Aulanier, G., \& Schmieder, B. 2002, A\&A, 386, 1106

Aulanier, G., Demoulin, P., van Driel-Gesztelyi, L., Mein, P., \& Deforest, C. 1998, A\&A, 335, 309

Aulanier, G., Demoulin, P., Mein, N., et al. 1999, A\&A, 342, 867

Aulanier, G., Srivastava, N., \& Martin, S. F. 2000, ApJ, 543, 447

Choe, G. S., \& Lee, L. C. 1992, Sol. Phys., 138, 291

Kuijpers, J. 1997, ApJ, 489, L20

Kuperus, M., \& Raadu, M. A. 1974, A\&A, 31, 189

Leenaarts, J., Rutten, R. J., Carlsson, M., \& Uitenbroek, H. 2006, A\&A, 452, 15

Lin, J., Forbes, T. G., \& Isenberg, P. A. 2001, JGR, 106, 25

Lin, Y., Wiik, J. E., Engvold, O., Rouppe van der Voort, L., \& Frank, Z. A. 2005, Sol. Phys., 227, 283

López Ariste, A., Aulanier, G., Schmieder, B., \& Sainz Dalda, A. 2006, A\&A, 456, 725

Mackay, D. H., Gaizauskas, V., \& van Ballegooijen, A. A. 2000, ApJ, 544, 1122

Mackay, D. H., \& van Ballegooijen, A. A. 2006, ApJ, 641, 577

Magara, T., \& Kitai, R. 1999, ApJ, 524, 469

Malherbe, J. M., \& Priest, E. 1983a, A\&A, 123, 80

Malherbe, J. M., Schmieder, B., Ribes, E., \& Mein, P. 1983b, A\&A, 119, 197

Martens, P. C., \& Zwaan, C. 2001, APJ, 558, 872

Martin, S. F., \& Echols, C. R. 1994, Solar Surface Magnetism, NATO Advanced Science Institutes (ASI) Series C: Mathematical and Physical Sciences, Proceedings of the NATO Advanced Research Workshop, held Soesterberg, the Netherlands, November 1-5, 1993, ed. R. J. Rutten, \& C. J. Schrijver (Dordrecht: Kluwer Academic Publishers), 339

Martin, S. F., Livi, S. H. B., \& Wang, J. 1985, Aust. J. Phys., 38, 929

Martres, M. J., Mein, P., Schmieder, B., \& Soru-Escaut, I. 1981, Sol. Phys., 69, 301

Molowny Horas, R. L. 1994, Thesis Institute of Theoretical Astrophysics University of Oslo

November, L. J., \& Simon, G. W. 1988, ApJ, 333, 427

Plocieniak, S., \& Rompolt, B. 1973, Sol. Phys., 29, 399

Priest, E. R. 1997, New Perspectives on Solar Prominences, proceedings of a meeting held in Aussois, France, 28 April-4 May 1997, ed. D. F. Webb, B. Schmieder, \& D. M. Rust, ASP Conf. Ser. 150, IAU Colloq., 167, 453

Ridgway, C., \& Priest, E. 1993, Sol. Phys., 146, 277

Rieutord, M., Roudier, T., Ludwig, H.-G., Nordlund, Å., \& Stein, R. 2001, A\&A, 377,14

Roudier, Th., Rieutord, M., Malherbe, J. M., \& Vigneau, J. 1999, A\&A, 349, 301

Rutten, R. J., Hammerschlag, R. H., Bettonvil, F. C. M., Sütterlin, P., \& de Wijn, A. G. 2004, A\&A, 413, 1183

Schmieder, B., Malherbe, J. M., Mein, P., \& Tandberg-Hanssen, E. 1984, A\&A, 136,81

Schmieder, B., Raadu, M. A., \& Wiik, J. E. 1991, A\&A, 252, 353

Schmieder, B., Aulanier, G., Main, P., \& Ariste, A. Lopez 2006, Sol. Phys., 238, 245

Van Ballegooijen, A. A. 2004, ApJ, 612, 519

Van Ballegooijen, A. A., \& Martens, P. C. H. 1989, ApJ, 343, 971 